\title{
New Proposal to Calculate the Friction in Sheet Metal Forming Through Bending Under Tension Test
}

\author{
Luis Folle $^{a * \bullet}$, Lírio Schaeffer ${ }^{b}$ \\ ${ }^{a}$ Centro Universitário Ritter dos Reis, Canoas, RS, Brasil \\ ${ }^{b}$ Universidade Federal do Rio Grande do Sul, Porto Alegre, RS, Brasil
}

Received: September 16, 2019; Revised: November 05, 2019; Accepted: November 10, 2019

\begin{abstract}
There are 6 different types of equations that have been formulated to measure friction through the bending under tension test, however, there is no work to show whether these calculations actually represent what is happening in a sheet metal stamping process. This paper aims to make a direct comparison between the bending under tension test and the sheet metal forming of a test piece to see if the friction coefficient reported by the test is able to predict friction in a real part. Several sources of information were used such as computer simulations, bending under tension test with different sensors and sheet metal forming tests of a cylindrical geometry to evaluate the friction. The results indicate that the equations already developed are not able to accurately predict the friction at the sheet interface and, therefore, a new equation was developed for this that is simpler to measure and presented satisfactory results.
\end{abstract}

Keywords: Friction coefficient, sheet metal forming process, bending under tension test, contact pressure.

\section{Introduction}

The bending under tension (BUT) test is currently the most commonly used test to simulate and isolate the friction generated in a stamping process. Several articles have already studied the subject and obtained as a result the value of friction. Sniekers and Smits ${ }^{1}$ were the first to introduce the idea of measuring torque to calculate friction, and for this purpose, they proposed a mathematical formulation that included torque in calculating the coefficient of friction. However, they only made a few measurements to find the friction value without worrying about whether it was correct. Nanayakkara et al. ${ }^{2}$ were the first researchers to compare three types of equations used to calculate friction in the BUT test, and to do so, also used the results to calculate friction for different pin sizes. However, they also did not bother to verify if that the results were correct. Berglund et al. ${ }^{3}$ used the BUT test to evaluate the correlation between punch material, machining finish and the friction coefficient result, however, they did not specify which equation was used for it and also did not worry if the friction was correct, only evaluated the difference between the values. In the work of Fratini et al. ${ }^{4}$ BUT test was used to measure friction when the pin is chromed or only machined with Teflon lubricant, grease and without lubrication and also the results were correlated only, that is, only the variation between values was investigated, not caring if the value itself was correct.

Another parameter that deserves attention is the contact pressure between the sheet and the tools, which in both the BUT test and the stamping of a part is difficult to measure. There are efforts to determine this pressure, but so far only the result given by numerical simulation is known.
Some researchers have found that the profile of this pressure is not constant, that is, there are two pressure peaks located near the beginning and end of contact between the sheet and the pin. This has been shown to occur in the same way as the sheet passes through the radius of the die. Sniekers and Smits ${ }^{1}$ demonstrated through the finite element simulation of the BUT test that this contact pressure between the sheet and the pin had an unequal profile over its entire length and that there were two major pressure peaks.

Kim et al. ${ }^{5}$ made a study, also through a finite elements simulation software, of the pressure generated between the pin and the sheet and found the same as Sniekers and Smits ${ }^{1}$. In addition, Kim et al. ${ }^{5}$ found that the angle of pressure acting on the pin is smaller than the bending angle of the die (90 degrees). Pereira et al. ${ }^{6}$ conducted an analytical study through finite element simulation of the evolution of contact pressure in the die radius during the stamping of a circular profile. They found the same pressure peaks that occur during bending of the sheet over the radius of the die and that this is due to high bending moments that occur in these regions causing the central part of these peaks to lose contact, greatly reducing the pressure in this region. Pereira et al. ${ }^{6}$ also concluded in their work that the contact angle between the sheet and the die is less than $90^{\circ}$. Pereira et al..$^{7}$ made a more elaborate study on the evolution of contact pressure of a straight strip of sheet in the region of the bending radius of the die. They demonstrated that there is a transient region in the contact pressure that corresponds to the beginning of sheet deformation, after which the pressure stabilizes. They also demonstrated that the parameters that most influence this pressure are the material yield stress and the relationship between the radius of the die and the thickness of the sheet. 
The results shown so far were all based on finite element numerical simulation, however, Coubrough et al. ${ }^{8}$ obtained results measured by the addition of a piezoelectric material film to the BUT test pin. The results obtained by them through this film were in Volts because this film had not been calibrated, however, this measurement was proportional to the pressure value in the pin. They performed two tests, one with the free pin (where the pin can rotate freely about its axis) and another with the static pin and proved what had been simulated by the authors mentioned above. They also observed that the beginning of the contact between the sheet and the pin occurred at an angle before the tangent that the sheet makes with the pin, which is in agreement with the simulations of Pereira et al. ${ }^{6}$.

Finally, Hoffmann et al. ${ }^{9}$ made a study where they compared the wear occurred in the die radius for 7 combinations of sheet and die material. They measured wear on the radius of the die and compared with numerical simulations. The results were that the highest wear occurred exactly in the regions where the contact pressure between sheet and die presented high values and that these regions of greater wear were located at the inlet and outlet of the bending angle of the die.

All the work shown until here has been concerned with some aspect of research into the phenomena that occur during sheet/die/lubrication interaction, but have not considered whether the BUT test actually generates friction values that are compatible with reality. Because of this, the purpose of this work is to verify this compatibility. Thus, several tests were made with the stamping of a single piece, as with the BUT test and compared with the simulation.

\section{Materials and Methods}

The material that was used in the present work is commercially pure aluminum Al 1100 and the properties are described in Table 1 . The coefficients $C$ and $n$ were obtained according to ISO 10275 : $2007^{10}$, anisotropy was obtained according to ISO 10113: $2006^{11}$ and the rest of the values were obtained through the tensile test through ISO 6892-1: 2016 ${ }^{12}$.
Table 1. Properties used to define the sheet material.

\begin{tabular}{lcc}
\hline \multicolumn{3}{c}{ Material Proprieties } \\
\hline Density & 2,7 & $\mathrm{~g} / \mathrm{cm}^{3}$ \\
Young's modulus & 69000 & $\mathrm{MPa}$ \\
Poisson ratio & 0,33 & \\
Strain hardening exponent $(\boldsymbol{n})$ & 0,09 & \\
Strain hardening coefficient $(\boldsymbol{C})$ & 196 & $\mathrm{MPa}$ \\
Anisotropy $\left(0^{\circ}\right)$ & 0,82 & \\
Anisotropy $\left(45^{\circ}\right)$ & 0,76 & \\
Anisotropy $\left(90^{\circ}\right)$ & 0,81 & \\
Yield strength & 124 & $\mathrm{MPa}$ \\
Yield strein & 0,2 & $\%$ \\
Thickness & 1 & $\mathrm{~mm}$ \\
\hline
\end{tabular}

\subsection{Bending under tension test}

The BUT test consists of bending a strip of sheet through a predetermined radius pin and sliding the sheet over it. To do this, a force is applied to one end of the sheet to provide relative movement between the sheet and the pin. At the other end a back tension force is applied to bend the sheet over the pin and the contact pressure on the pin can be varied. Figure 1 shows a schematic drawing of this test. The force that generates the movement is called the drawing force (F1 in Figure 1) and the force that is applied in the opposite direction is the back tension force (F2 in Figure 1). The radius pin $r$ has the function of simulating the friction in the passage of the die radius, since in this region that the tensions are higher.

In this test, there are two forces required to make the sheet slide over the pin, one is the frictional force between the contact surfaces and the other is the force required to perform sheet bending and unbending. Since the purpose of the test is to know the frictional force between the contact surfaces, it is performed in two steps. In the first one, the pin through which the sheet passes, can freely rotate through its axis, so that there is no relative movement in the pin/sheet interface.

\section{A: Free Rotating Pin}

\section{B: Fixed Pin}

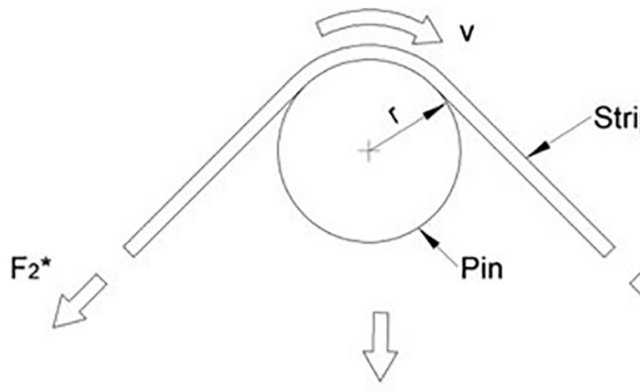

Vertical Force

Acting on the Pin

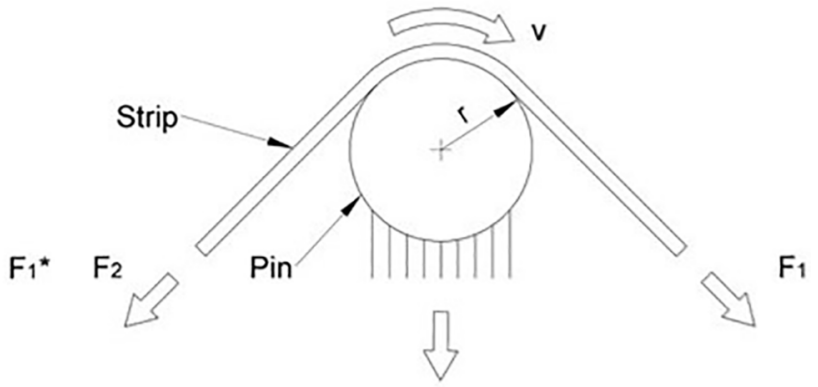

Vertical Force

Acting on the Pin

Figure 1. Schematic figure of the BUT test. Source: based on Andreasen et al. ${ }^{13}$ 
This generates a minimum friction condition, and the force required to make the sheet move is due only to the bending and unbending force of the sheet. In the second step, this same pin is fixed to its axis preventing any movement. The force required to make the sheet move is then the bending force plus the frictional force. Thus, the bending force measured in the first stage can be discounted of the second stage and only the frictional force obtained.

The machine used for friction measurement, see Figure 2, was designed to measure and evaluate the friction coefficient in stamping process and is based on the BUT test. For this, there are two hydraulic cylinders which have the function of sliding a strip of metal over a pin. These hydraulic cylinders were mounted in a triangular structure that aims to decrease the influence of vibration giving more stability to transmit forces during the test. On the upper vertex of the machine there is a pin that can be supported with or without bearings, see figure 2 . Over this pin goes the sheet metal strip with $90^{\circ}$ bending angle.

On the left side of the machine, there is a load cell coupled to the hydraulic cylinder, which has the role of measuring the drawing force on the sheet. On the right side of the machine, there is another load cell that has the function of measuring the back tension force acting on the sheet. Coupled to the pin through which the sheet passes there is a torque sensor, which aims to measure the torque to which the pin is subjected when the sheet passes through it. Below the pin, there is also a load cell that aims to measure the vertical force on it.

\subsection{Stamping test}

Since the aim of this study is to measure the friction coefficient in sheet metal stamping through the bending under tension test, a stamping test was performed to measure the parameters that would be later compared with a numerical simulation to find the friction coefficient that governs the process. This test was based on work of Kim et al. ${ }^{14}$. For that, a simple geometry was stamped and the force and displacement of the punch were measured as a comparison with the simulation results. Basically the tool set used for the test is composed of a cylindrical punch, blank-holder and die. Dimensions are shown in Figure 3. Cylindrical specimens that are constrained by the blank-holder were used. The punch is then forced against the blank causing the stamping, that is, the sheet is forced into the die until the material is stamped.
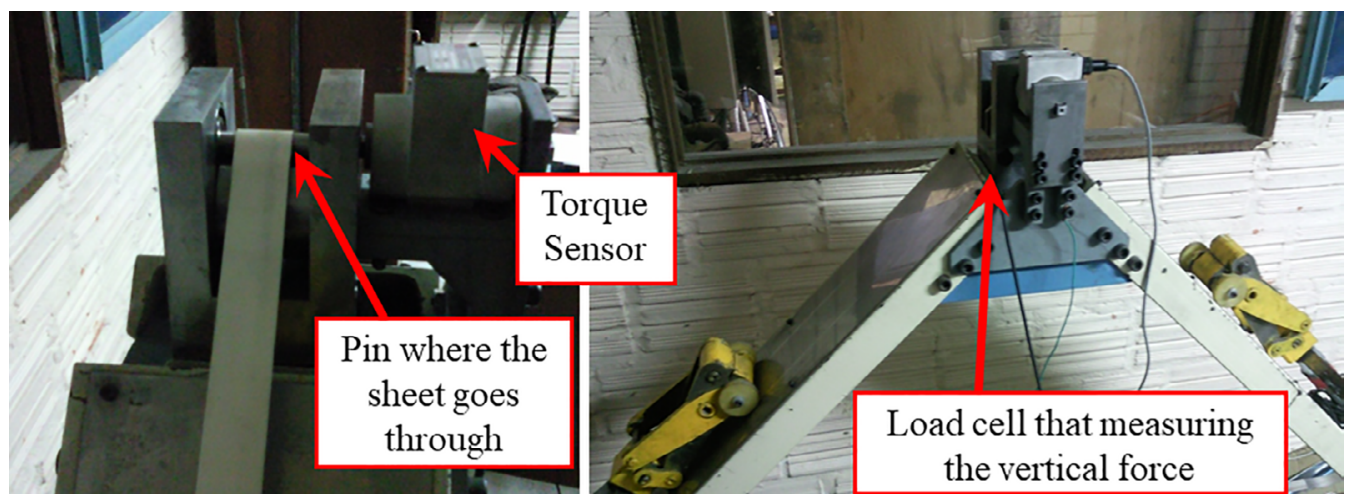

Figure 2. Machine used to perform BUT test.

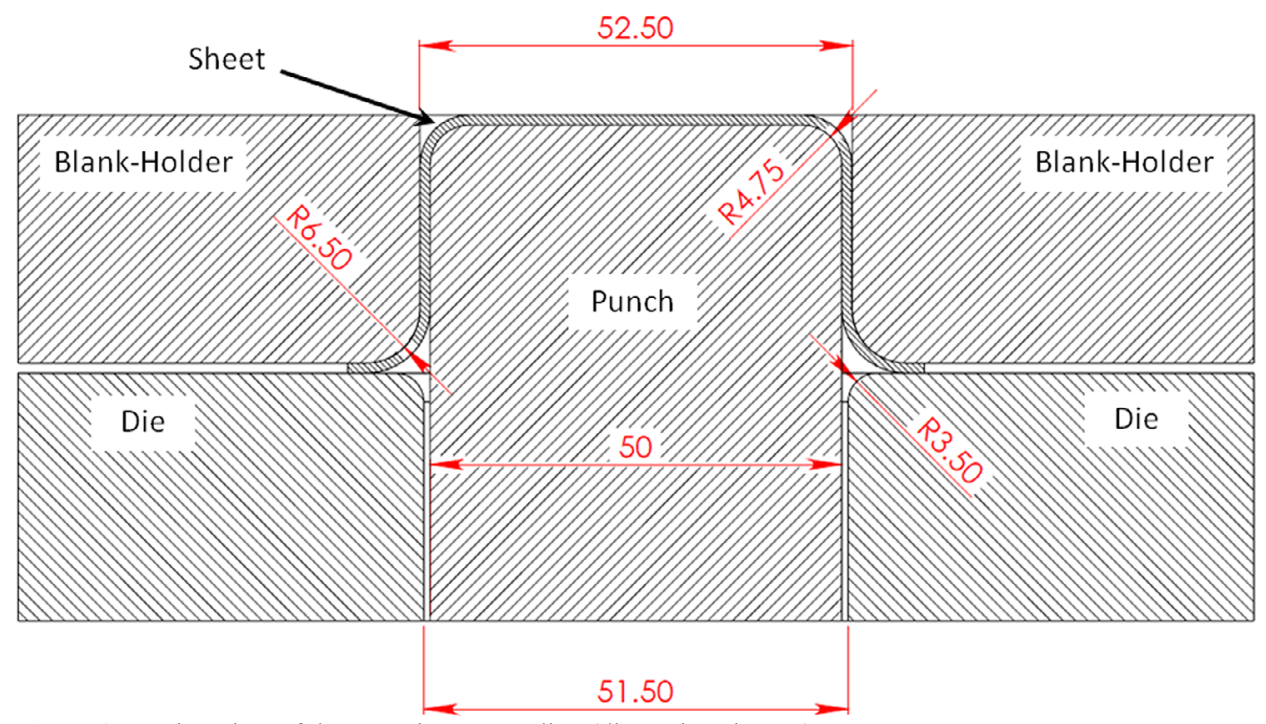

Figure 3. Section view of the stamping test tooling (dimensions in $\mathrm{mm}$ ). 
The surface roughness parameters Ra and Rt of the tools and sheet material used in this study are described in Table 2.

The lubricants used for the stamping tests were a $0.09 \mathrm{~mm}$ thick Teflon sheet. That lubricant was selected because based on the work of Fratini et al. ${ }^{4}$, it was the lubricant that generated the lowest measured friction. This is important to evaluate the friction in the simulation as it should be low enough to compare with the BUT test made with a polymeric sensor which will be explained below.

Table 2. Surface roughness parameters of tools and sheet material in $\mu \mathrm{m}$.

\begin{tabular}{lcc}
\hline \multicolumn{1}{c}{ ROUGHNESS } & RA & RT \\
\hline Blank-holder & 0.20 & 2.36 \\
Die & 0.30 & 2.38 \\
Punch & 0.11 & 1.46 \\
Sheet & 0.36 & 3.79 \\
\hline
\end{tabular}

\subsection{Actual contact pressure measurement}

The contact pressure between tools and the sheet is difficult to measure, however, it influences the friction results between the contacting surfaces. For this, a sensor called Pressurex ${ }^{\circledR}$ was used which is marketed by Sensor Products Inc.

Pressurex $®$ is a mylar (polyester) based film that contains a layer of micro capsules. Applying force to the film causes the micro capsules to rupture, producing a high-resolution instantaneous and permanent topographic image of pressure variation across the contact area (Figure 4). Pressurex ${ }^{\circledR}$ color intensity is directly related to the amount of pressure applied to it. The higher the pressure, the more intense the color will be. The numerical value of the pressure is evaluated by a color intensity standard that is provided by the manufacturer (see Figure 5). Pressurex ${ }^{\circledR}$ film is 0.1 to $0.2 \mathrm{~mm}$ thick, allowing it to be applied to curved surfaces as well. According to the film manufacturer the measurement inaccuracy is of the order of $10 \%$.
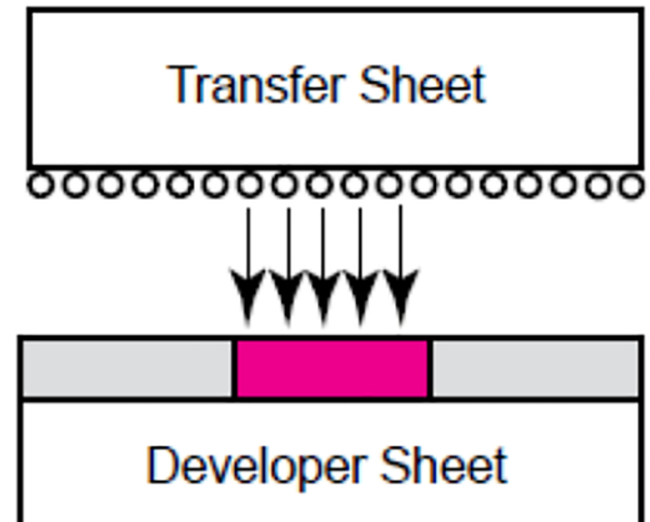

Figure 4. Cross-sectional view of Pressurex ${ }^{\circledR}$ film.
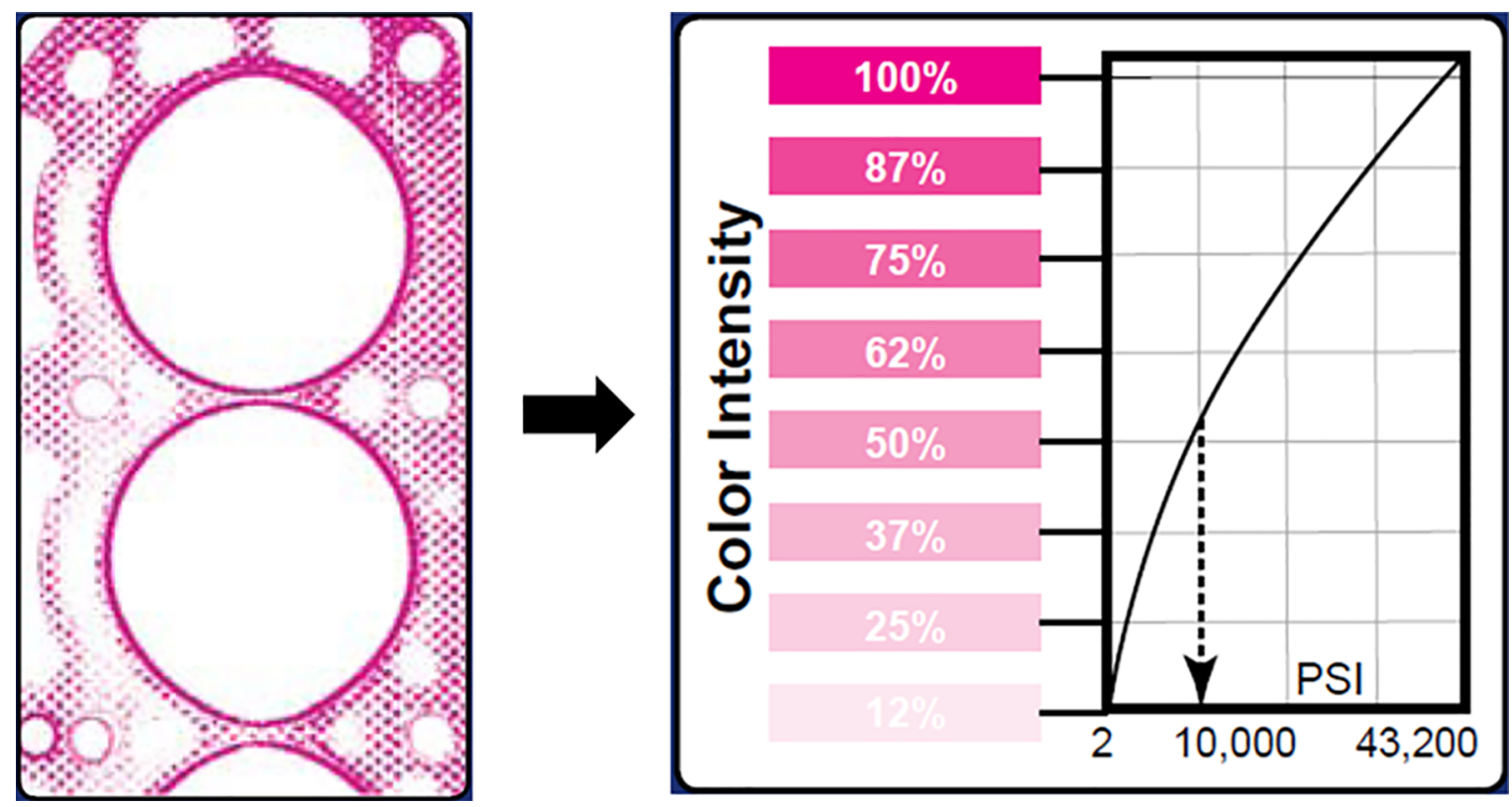

Substrate (polyester base) thickness (4 mils) Microcapsule layer

\section{_ Color developing layer Substrate — (polyester base) thickness (4 mils)}

Figure 5. Pressure measurement through Pressurex ${ }^{\circledR}$. 
This sensor was used in both the BUT test and the stamping test to measure the contact pressure acting on the pin in the BUT test. This was done to compare with the pressure measured by the vertical load cell and also with the pressure exerted on the sheet during the stamping test.

\subsection{Simulation method}

The specific objective of the numerical simulation is to reproduce the experimental study, thus allowing to evaluate the coefficient of friction and to compare the force versus displacement values of the punch with the measured results.

For the analysis of the desired geometry the DYNAFORM Version 5.6 simulation software with the LS-Dyna version 971 solver was used.

The elements used to define the blank material and tools (punch, die and blank-holder) are shell type with five integration points in thickness, with blank being considered as an elastoplastic material according to the Ludwig-Hollomon model and the tools as undeformable. The mesh used in the tools was $2 \mathrm{~mm}$ with $0.5 \mathrm{~mm}$ in the rounding region and $0.5 \mathrm{~mm}$ in the blank where results converged. The friction model used was that of Coulomb and the type of contact (Contact interface) was that of "Form one way Surface to Surface". Figure 6 shows the construction of the entire assembly mounted on the simulator for stamping with the generated meshes and details of mesh refinement in the critical regions.

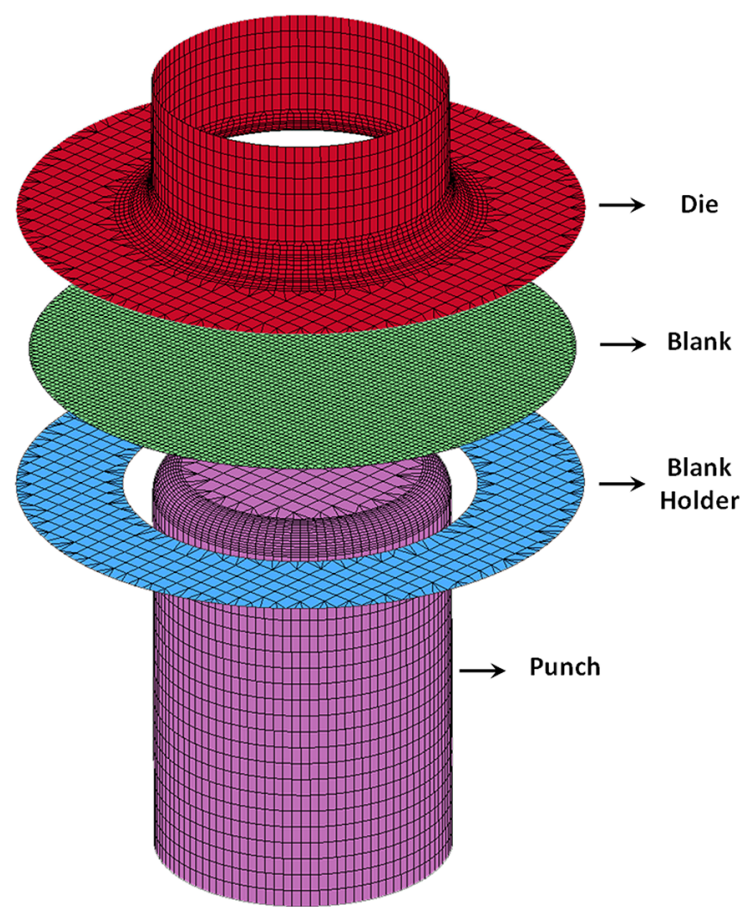

Figure 6. Assembly made in DYNAFORM to simulate the stamping process used.
The simulation was divided into three steps: in the first, the blank-holder moves until it reaches the sheet; in the second, the blank-holder applies a force of $10 \mathrm{kN}$, and in the third, the punch is displaced $27 \mathrm{~mm}$, causing almost the entire blank to be stamped. This value was specified as the displacement obtained in the experimental tests.

\subsection{Equations used to calculate the coefficient of friction}

The equations that are used to determine friction using the BUT test have been modified to be more accurate in calculating friction. The first approximation for calculating the coefficient of friction was based on the equations for calculating pulleys. In this case, friction at the pin/sheet interface is given by a natural logarithm of the ratio between drawing force and back tension force as can be seen in equation (1).

$$
\mu=\frac{2}{\pi} \ln \frac{F_{1}}{F_{2}}
$$

Where $\boldsymbol{F}_{1}$ is the drawing force and $\boldsymbol{F}_{2}$ is the back tension force. The term $2 / \pi$ refers to the 90 -degree angle between forces F1 and F2. However, as mentioned above, it is necessary to perform two tests so that the actuation of the bending and unbending force of the sheet can be discounted. Therefore, in equation (1) a term was added that refers to the bending force of the sheet. This term is called the bending force $\boldsymbol{F}_{b}$ of equation (2) and is obtained by subtracting the drawing and back tension forces during the free-pin test, $\boldsymbol{F}_{1}{ }^{*}$ and $\boldsymbol{F}_{2}{ }^{*}$ of equation (3), respectively.

$$
\begin{gathered}
\mu=\frac{2}{\pi} \ln \frac{\left(F_{1}-F_{b}\right)}{F_{2}} \\
F_{b}=F_{1}^{*}-F_{2}^{*}
\end{gathered}
$$

As can be seen from equations (1) and (2), the test geometry, pin radius and sheet thickness have no contribution to friction. Therefore, another way of measuring the coefficient of friction was proposed where the radius of the pin and the sheet thickness are taken into account, equation (4). This equation was used in the works of Han ${ }^{15}$, Jonasson et al. ${ }^{16}$, Wihlborg and Gunnarsson ${ }^{17}$, Nanayakkara et al. ${ }^{2}$.

$$
\mu=\frac{2}{\pi}\left[\frac{R+0.5 t}{R}\right] \ln \left[\frac{F_{1}-F_{b}}{F_{2}}\right]
$$

Where $\boldsymbol{t}$ is the thickness of the sheet and $\boldsymbol{R}$ is the radius of the pin.

It may be noted that all the equations mentioned so far are composed of a natural logarithm of the ratio of two or more forces. However, another way to calculate friction was proposed, which can be seen in equation (5). 
This equation has been described and used by Saha et al. ${ }^{18}$ and Fratini et al. ${ }^{4}$.

$$
\mu=\frac{2 \cdot\left(F_{1}-F_{2}-F_{b}\right)}{\Theta \cdot\left(F_{1}+F_{2}\right)}
$$

Where $\boldsymbol{F}_{\boldsymbol{I}}$ is the drawing force, $\boldsymbol{F}_{2}$ is the back tension force, $\boldsymbol{F}_{\boldsymbol{b}}$ is the bending force and $\Theta$ is the bending angle of the sheet.

As mentioned earlier, Sniekers and Smits ${ }^{1}$ and later Andreasen et al. ${ }^{13}$ used a torque sensor on the BUT test pin to eliminate the second step of the free pin test. Equation (6), conceived by Sniekers and Smits ${ }^{1}$, shows how the coefficient of friction is calculated by measuring a torque.

$$
\mu=\frac{\frac{F_{0} d}{R}}{\sqrt{F_{1}^{2}+F_{2}^{2}-\left(\frac{F_{0} d}{R}\right)^{2}}}
$$

Where the term $\boldsymbol{F}_{\boldsymbol{0}} \boldsymbol{d}$ represents the torque on the pin, $\boldsymbol{F}_{\boldsymbol{1}}$ is the drawing force and $\boldsymbol{F}_{2}$ is the back tension force.

The equation of Andreasen et al. ${ }^{13}$, was designed to know the frictional stress that occurs at the pin/sheet interface and is given by equation (7).

$$
\tau=\frac{2 T}{\pi W R^{2}}
$$

Where $\tau$ is the frictional stress, $\boldsymbol{T}$ represents the pin torque, $\boldsymbol{W}$ is the width of the sheet strip passing over the pin and $\boldsymbol{R}$ is the radius of the pin.

It can be seen that for the calculation of the coefficient of friction several forms have been proposed, but for the calculation of the contact pressure between the sheet and the pin, almost all authors use the same equation (8).

$$
p=\frac{F_{1}+F_{2}}{2 W R}
$$

Where $\boldsymbol{p}$ is the contact pressure, $\boldsymbol{F}_{\boldsymbol{I}}$ is the drawing force, $\boldsymbol{F}_{2}$ is the back tension force, $\boldsymbol{W}$ is the width of the sheet that passes over the pin, and $\boldsymbol{R}$ is the radius of the pin.

The relationship between equation (7) and (8) generates the coefficient of friction, equation (9).

$$
\mu=\frac{\tau}{p}=\frac{4 T}{\pi R\left(F_{1}+F_{2}\right)}
$$

Contact pressure that is calculated by equation (8) according to the authors $\operatorname{Han}^{15}$, Jonasson et al. ${ }^{16}$, Hao et al. ${ }^{19}$, Wihlborg and Gunnarsson ${ }^{17}$, Kim et al. ${ }^{5}$, Andreasen et al. ${ }^{13}$, Miguel et $\mathrm{al}^{20}$ is the most common. However, a variation of this expression is shown in equation (10) that was proposed by Wilson in 1996, obtained from the work Fratini et al. ${ }^{4}$.

$$
P=\frac{F_{1}+F_{2}}{2 W R} \operatorname{sen} \frac{\theta}{2}
$$

There is also a third variation of equation (8) that was proposed by Sube ${ }^{21}$ and is shown in equation (11).

$$
P=\frac{\left(F_{1}+F_{2}\right)-F_{b}}{2 W R}
$$

All of the above equations make use of the drawing and back tension forces and equally do not measure the vertical force that act on the pin. Due to this, a new equation will be proposed here, considering only the vertical force and the torque (12).

$$
\mu=k \frac{F_{V}}{T R}
$$

Where $\boldsymbol{k}$ is an adjustment coefficient, $\boldsymbol{F}_{\boldsymbol{V}}$ is the vertical force on the pin, $\boldsymbol{T}$ represents the pin torque and $\boldsymbol{R}$ is the radius of the pin.

\section{Results and Discussions}

\subsection{Comparison of results by stamping test with simulation}

The graph resulting from the measurement of the force on the punch by its displacement and the numerical simulation are shown in Figure 7. The numerical simulation of the stamping test was done using DYNAFORM where the friction coefficient adopted for each simulation was arbitrated until the curve of simulated force and displacement would fit the stamping test. For this, it was assumed that the Teflon sheet would generate very low friction and thus the coefficient of friction placed in the simulation would have to be low. In the study by Fratini et al. ${ }^{4}$, the values of the coefficient of friction were between 0.09 and 0.01 . Among all simulations performed, the only parameter that was evaluated was the coefficient of friction, all other constants remaining constant. Figure 7 shows the comparison between the measured force and displacement result for the Teflon sheet and the simulation with a coefficient of friction of 0.056 .

Friction Coefficient of 0,056

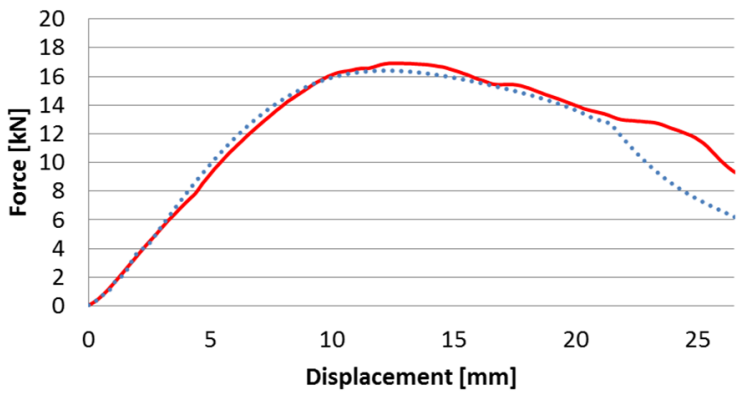

Measured with teflon …... Simulated

Figure 7. Comparison between the graphs generated by the simulation and those measured with teflon. 
By analyzing the graphs in Figure 7 it is possible to observe that the curves practically fit each other. They deviate after $20 \mathrm{~mm}$ which corresponds to the region where the thickness increases in the stamping test due to the accumulation of deformations and which the simulation cannot predict because it considers the sheet to be a surface without volume. It is important to note that the BUT test should give a coefficient of friction of 0.056 if the same conditions as the stamping test are used, this also includes a contact pressure close to the actual test.

\subsection{Contact pressure measurement by pressure sensor}

Through the Pressurex ${ }^{\circledR}$ films it was possible to evaluate the contact pressure intensity in both stamped geometry and sheet metal strip during the BUT test. Figure 8 shows the application of Pressurex ${ }^{\circledR}$ film in three stages of the stamped geometry deformation where the highest pressure was obtained. Since the pressure film acts under static conditions, the pressure had to be measured with a minimum of slip between the sheet and the die, so preforming the sheet was done before placing the film itself. Figure 9 shows the result of applying Pressurex ${ }^{\circledR}$ films to the strip during the BUT test. The pressure film was measured only for the most deformed part (Figure 8a), since the part at this stage reached the maximum stamping force and corresponds to the maximum stress in the region of the radius of the die.
To get a clearer idea of the influence of this contact pressure for different sheet stress levels, measurements were made with the Pressurex ${ }^{\circledR}$ film on the BUT test pin for 3 pressure levels. Figure 10 shows the force on each side of the sheet for these 3 pressures, called "Maximum, Average and Minimum". Tests were made with the fixed pin and after with the free pin. The "Maximum" level corresponds to the pressure that is exerted on the sheet very close to its rupture.

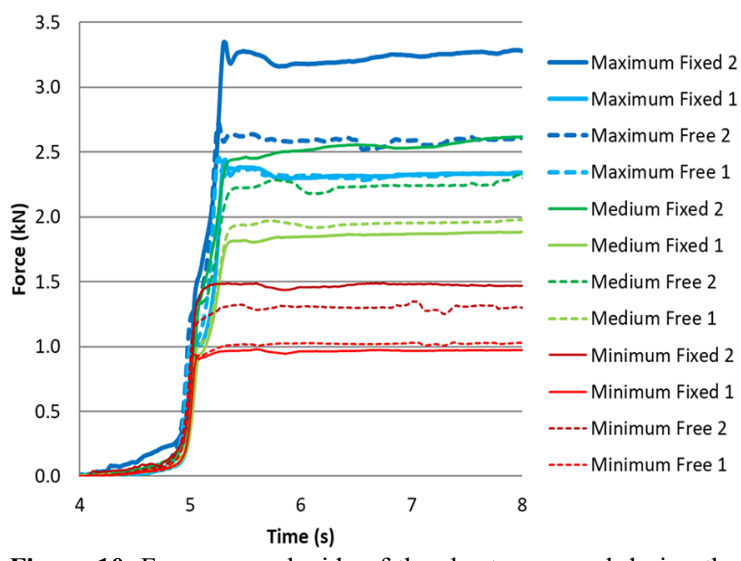

Figure 10. Force on each side of the sheet measured during the BUT test. The number 2 in the legend description means drawing force and the number 1 means back tension force.

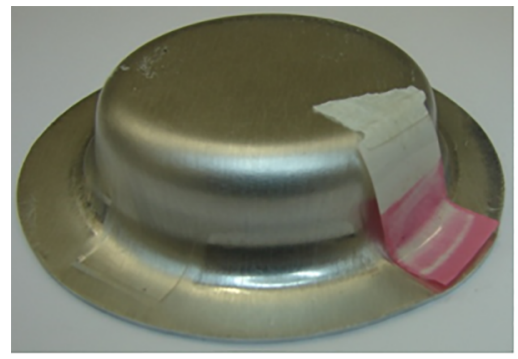

(a)

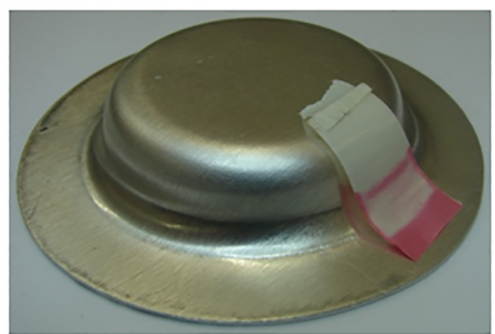

(b)

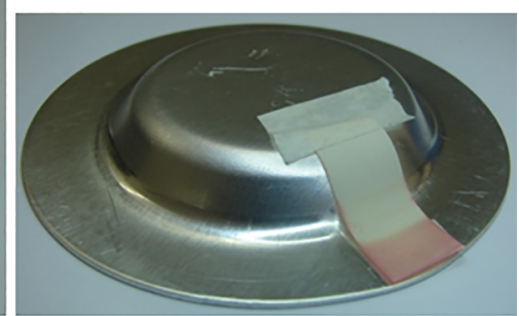

(c)

Figure 8. Application of Pressurex ${ }^{\circledR}$ film in three stages of part deformation, (a) $20 \mathrm{~mm}$ punch stroke, (b) $15 \mathrm{~mm}$ punch stroke, (c) $10 \mathrm{~mm}$ punch stroke.

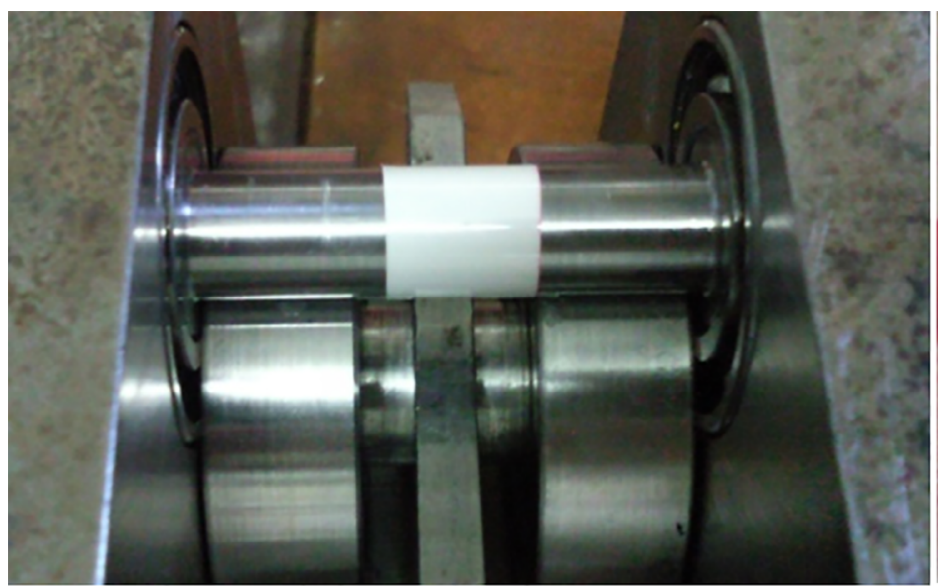

(a)

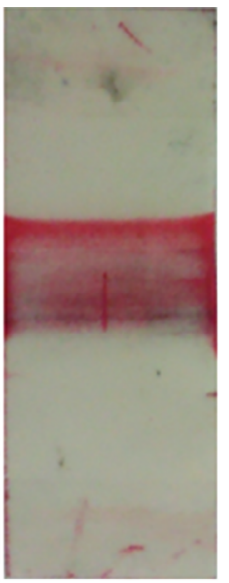

(b)

Figure 9. Pressurex ${ }^{\circledR}$ film applied to the strip during the BUT test (a) and after-test films (b). 
The "Minimum" level corresponds to the minimum pressure value the machine applies to the sheet, that is, only the force required to move the hydraulic cylinder without loading. And finally, the level "Medium" corresponds to an intermediate value between the maximum and the minimum. From the curves of Figure 10 it can be seen that when the pin is freely rotating, undetermined in pressure, the difference remains constant and that when the pin is fixed only the drawing force increases. In Figure 11, the torque value increases proportionally with increasing pressures.

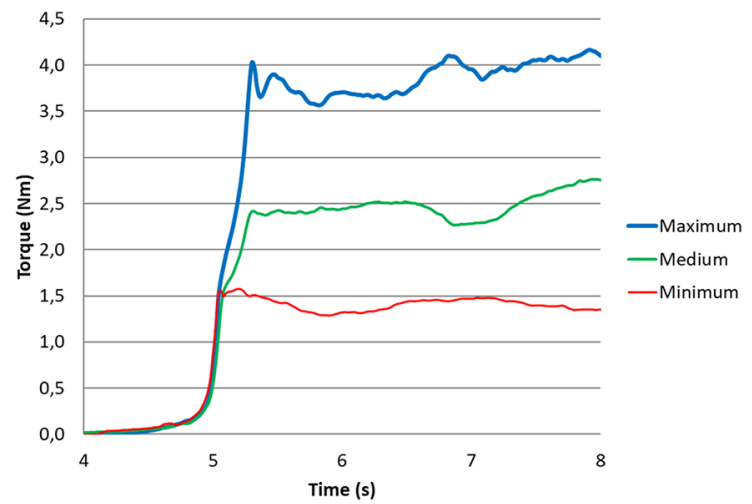

Figure 11. Torque measured over the pin during the BUT test.

As one of the objectives of this work is to evaluate the contact pressure acting on the BUT test, the vertical force acting on the pin was obtained both when it was fixed and when it was free. The contact pressure is then given by dividing the vertical force acting on the sheet by the area of action of that force. Figure 12 shows the vertical force measured for each pressure level. Note that the greater the forces acting on each side of the sheet, the greater the vertical force.

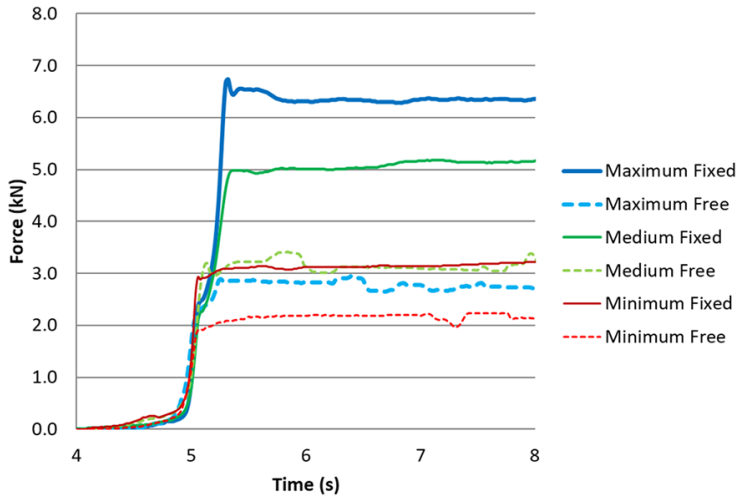

Figure 12. Vertical force measured on the pin during the BUT test.

It is also possible to notice that in the measurements with the free pin, the curves of the pressure "Medium" and the pressure "Maximum" were very close, being inverted of position. This is because the load cell that measures this force makes an average measurement of the pressures acting on the sheet, that is, there are pressure peaks that influence the average of the curve. This is evident in Figure 13, which are the Pressurex ${ }^{\circledR}$ films obtained in these tests. In the case of the measurement of the "Medium" pressure and the "Maximum" pressure for the free pin, it is possible to observe that the color, and therefore the pressure, are similar, being in the order of $28 \mathrm{MPa}$ each. From figure 13, it is also possible to observe that for measurements with the fixed pin, there are two characteristic peaks at the beginning and end of the sheet bending angle, which is in agreement with the studies of several authors, described at the beginning of this work.

Figure 13 shows the result of pressure film images, that is, qualitatively, but in figure 14, these results are shown in quantitative graphical form of the mean pressure values.
Maximum
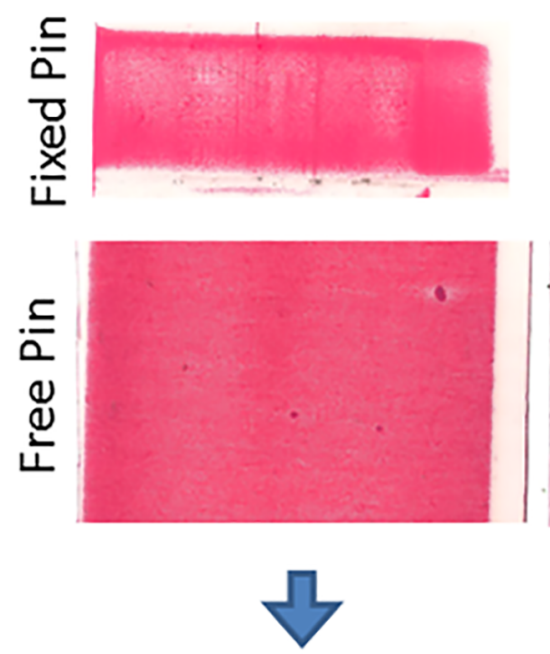

Medium
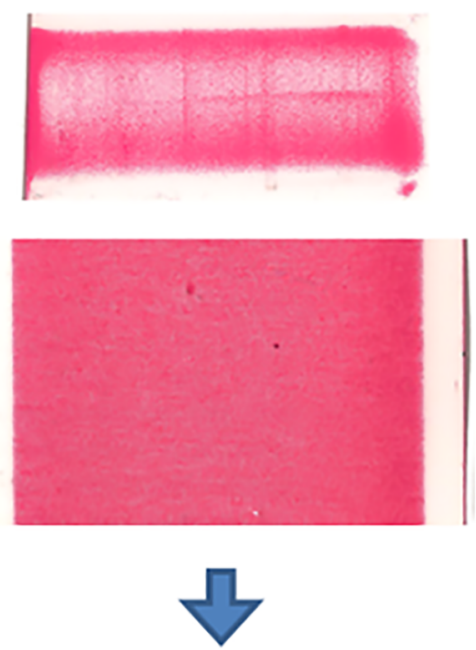

Minimum
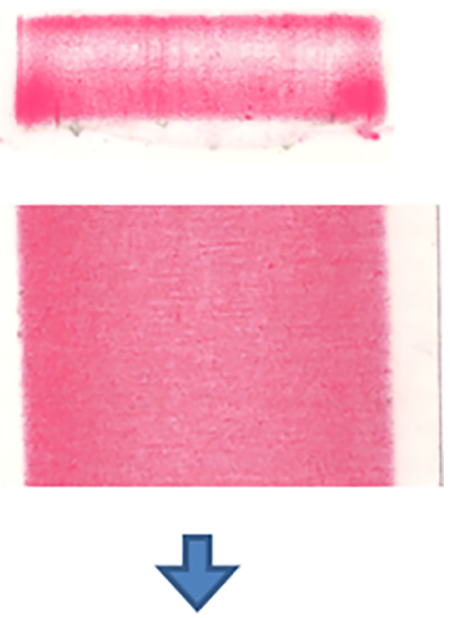

Direction of movement

Figure 13. Pressurex ${ }^{\circledR}$ film obtained for each pressure level with fixed and free pin. 


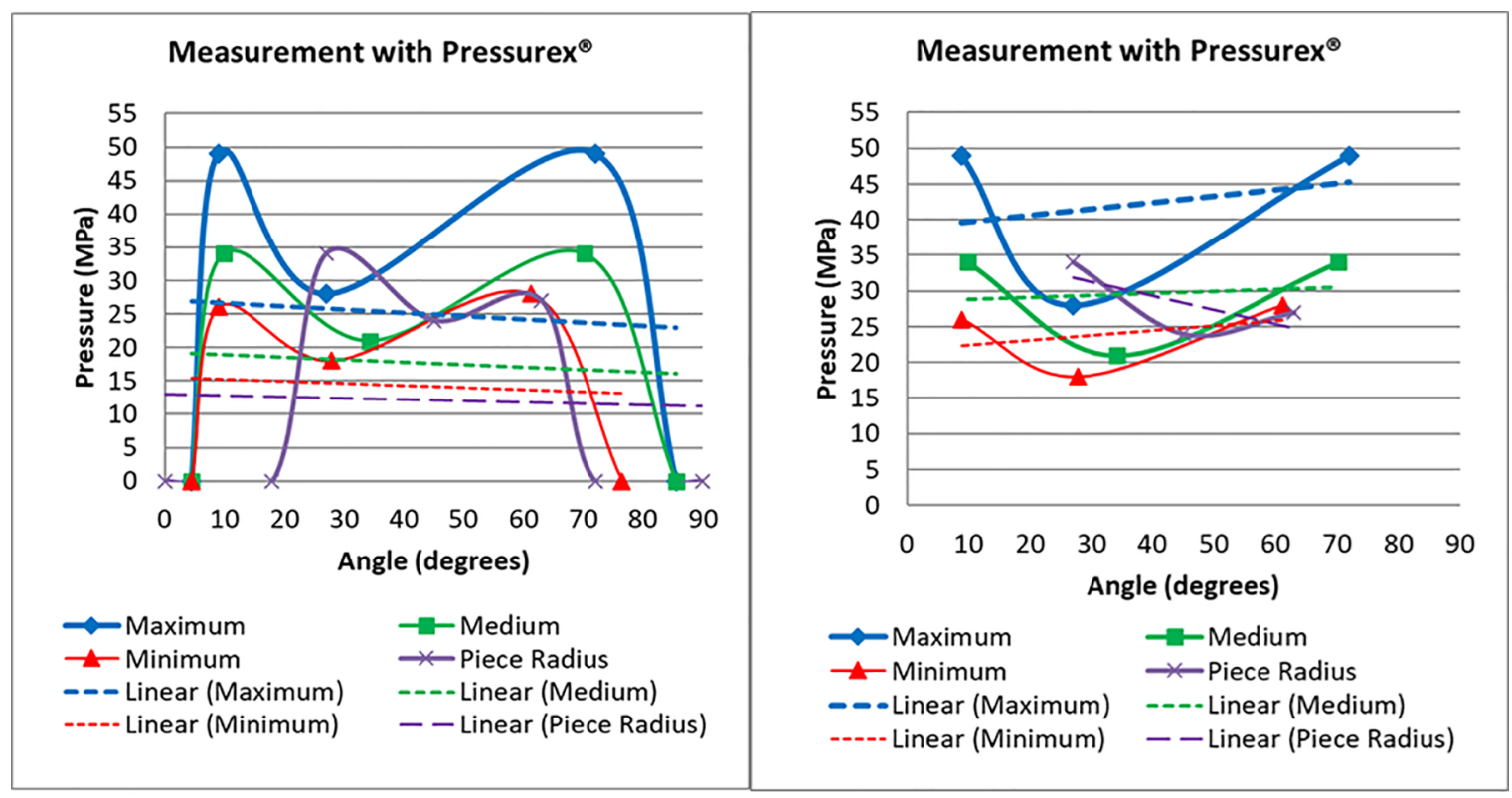

(a)

(b)

Figure 14. Pressure results obtained from Pressurex ${ }^{\circledR}$ films when the pin is fixed. Graph (a) shows the values considering the starting and ending points starting at zero and in graph (b) these points are removed.

From these graphs it is evident that there are very pronounced contact pressure peaks in both the bending radius of the sheet in the stamping test and the BUT test pin. The dashed lines on both graphs show the linear mean of these pressure peaks. This was done to compare with the results given by the vertical load cell as it measures a constant linear force and the profile of the pin pressures is quite variable. The graph in Figure 14a shows the result when the pressure starts and ends from zero and the graph in Figure 14b shows what is the linear mean when the start and end points are removed. In this circumstance (Figure 14b) the linear pressure averages are higher than in Figure 14a.

The first important observation from the graph in Figure 14 is that the pressure level reached at the workpiece radius in the stamping test is around $30 \mathrm{MPa}$. Another observation is that the action of this pressure does not occur in the entire bending angle, $90^{\circ}$, it acts in an internal region at this angle, and for the stamping test, the pressure acted in an even smaller region than in the BUT test. This is because in the stamping of the part, there is a gap between the punch and the die, causing the sheet not to bend at an angle of $90^{\circ}$, but smaller, further reducing the pressure actuation. This generates an error when calculating the contact pressure, since equations (8), (10) and (11) consider the pressure acting area to be covered by the entire bending angle, which was not verified. These results had already been discovered through previous studies that were shown at the beginning of this work, but no direct measurement of the value of this pressure had been made, the maximum that was obtained was a result of voltage in Volts as in the case of Coubrough's. et al. ${ }^{8}$ work and several numerical simulations of this test.
Table 3 shows the results of pin-sheet contact pressure for each pressure level through equations (8), (10) and (11). It was also made the calculation of the vertical force divided by the real area and two situations that correspond to the estimated pressure with zero and the estimated without zero. The pressures estimated with zero were obtained from figure 14a through the linearization of the curve points considering the starting and ending points zero. The estimated pressures without zero were obtained from figure $14 \mathrm{~b}$ through the linearization of curve points without the start and end points zero. It is important to note that if the starting and ending points zero are considered, the pressure given by the vertical force approximates the pressures measured by the pressure film. Since the "Medium" level curve showed a mean pressure very close to the pressure measured in the stamping test, this pressure level was considered to evaluate the coefficient of friction generated in the BUT test.

By measuring torque and forces on each side of the sheet it is possible to calculate the coefficient of friction for each equation described above. Table 4 shows these results. It can be observed that the coefficient of friction presents very close values regardless of the pressure level applied, however, as shown by the numerical simulation, the friction generated between the part and the matrix, when applying a polymeric interface should be around 0.056. It is important to note that Pressurex ${ }^{\circledR}$ film acts as an interface element separating the two surfaces, that is, a solid dry lubricant, as the film must be used with clean and dry surfaces. In other words, Pressurex ${ }^{\circledR}$ film maintains the ratio of frictional force to normal force almost the same regardless of the pressure that will be applied. 
Table 3. Contact pressure for several different calculation forms. Values in MPa.

\begin{tabular}{lcccccc}
\hline Pressure Levels & Equation (8) & Equation (10) & Equation (11) & $\begin{array}{c}\text { Vertical Force } \\
\text { with Real Area }\end{array}$ & $\begin{array}{c}\text { Estimated with } \\
\text { zero }\end{array}$ & $\begin{array}{c}\text { Estimated } \\
\text { without zero }\end{array}$ \\
\hline Maximum & 14.3 & 10.1 & 13.6 & 23.5 & 24.0 & 40.0 \\
Medium & 11.3 & 8.0 & 10.6 & 18.8 & 18.0 & 30.0 \\
Minimum & 6.3 & 4.4 & 5.5 & 13.1 & 14.0 & 22.0 \\
\hline
\end{tabular}

Table 4. Coefficient of friction calculated by different equations.

\begin{tabular}{lccccccc}
\hline \multicolumn{1}{c}{ Pressure Levels } & $\mathrm{Eq}(1)$ & $\mathrm{Eq}(2)$ & $\mathrm{Eq}(4)$ & $\mathrm{Eq}(5)$ & $\mathrm{Eq}(9)$ & $\mathrm{Eq} \mathrm{(6)}$ & $\mathrm{Eq} \mathrm{(12)}$ \\
\hline Maximum & 0.213 & 0.159 & 0.171 & 0.151 & 0.139 & 0.154 & 0.071 \\
Medium & 0.201 & 0.124 & 0.134 & 0.116 & 0.111 & 0.122 & 0.056 \\
Minimum & 0.267 & 0.131 & 0.141 & 0.115 & 0.113 & 0.124 & 0.051 \\
\hline
\end{tabular}

This is the expected behavior of a good performing lubricant where the lubrication layer does not break under pressure. This indicates that for a lubricant to perform well, it must not only lower the friction value but also keep it constant during the forming process. Equation (1) has higher coefficients of friction because it considers the bending force of the sheet. Thus, equation (1) should not be used for the purpose of calculating the coefficient of friction. The other equations are close but still very high compared to the 0.056 friction obtained with the numerical simulation. For this, a new equation was proposed that considered only the vertical force of the pin in relation to the torque (equation 12), where the coefficient of adjustment was determined to be 0.75 to fit the pressure level "Medium". The value of the coefficient of adjustment is given by 2 factors, the first is that the vertical force measured by the sensor is an average response to a pressure located on the sheet that is not uniform, that is, it has two peaks at the ends of the pin and a value smaller in the middle. The second factor is related to the first and is due to the area of action of this contact pressure that is smaller for the sheet and larger in the pin as can be seen in Figure 14a.

It is important to note that the difference between the coefficient of friction obtained by the simulation (0.056) and the lowest coefficient of friction given by the equations used so far (equation 9) is $50 \%$, which generates a very different result from the real one, being the equation (12) more realistic. However, a difficulty with the BUT test will always be determining which pressure level corresponds to an actual sheet metal forming. But even with this difficulty the difference between the "Maximum" and "Medium" pressure levels in relation to the coefficient of friction reached about $20 \%$, which is smaller than the $50 \%$ difference in relation to equation (9), which already enables the use of the proposed equation. Another important contribution of equation (12) is to make the test simpler since only 2 sensors would be needed on the machine and without the need to perform the free pin test, saving test samples.
However, these results are valid only for the material and geometry evaluated. To confirm that this equation is effective, it would be necessary to evaluate this method for other materials and lubrication conditions, as well as for other geometries such as sheet bending radii of different sizes.

\section{Conclusions}

This study aimed to propose a new equation for the calculation of the coefficient of friction from the BUT test and in this regard, some conclusions can be made.

Regarding the contact pressure measurement with the Pressurex ${ }^{\circledR}$ film, the film proved to be efficient to predict the pressures associated with the sheet contact with the pin, which is in full agreement with the results obtained by other authors. Measurement of contact pressure through the Pressurex ${ }^{\circledR}$ film showed that the vertical force on the pin is closer to actual values than the equations used so far. Another important contribution is that the pressure film showed the exact area of action of the sheet on the pin and that it will always be smaller than the geometrically calculated area regardless of the applied pressure level.

Regarding the proposed new equation, it was found that, with an adjustment coefficient of 0.75 , the BUT test for the "Medium" pressure level generated the same result compared to the simulation and that for other pressure levels the maximum difference was $20 \%$, below the difference from the other equations. Another observation was that the coefficient of friction remained close for the various equations at the three pressure levels. This suggests that a high performance lubricant is not necessarily one that generates the lowest friction levels, but one that can keep friction constant with varying pressure applied.

This paper aims to take a first step in obtaining $\mathrm{K}$ values. However, further study is needed to arrive at $\mathrm{K}$ values that are best suited for certain types of materials, finishes and lubricants, or perhaps a universal $\mathrm{K}$ value that fits any case. 
It is also important to note that the comparisons made in this study were between a cylindrical drawpiece and a straight pin, which probably generates a different stress state due to the curvature of the bending radius of the die in relation to the pin. Thus, the data presented in this article are only approximate, requiring more tests to be done to validate the proposed method.

\section{References}

1. Sniekers RJJM, Smits HAA. Experimental set-up and data processing of the radial strip-drawing friction test. Journal of Materials Processing Technology. 1997;66(1-3):216-223.

2. Nanayakkara NKBMP, Kelly G, Hodgson P. Application of bending under tension test to determine the effect of tool radius and the contact pressure on the coefficient of friction in sheet metal forming. Materials Forum. 2005;29(1):114-118.

3. Berglund J, Brown CA, Rosén BG, Bay N. Milled die steel surface roughness correlation with steel sheet friction. CIRP Annals - Manufacturing Technology. 2010;59(1):577-580.

4. Fratini L, Lo Casto S, Lo Valvo E. A technical note on an experimental device to measure friction coefficient in sheet metal forming. Journal of Materials Processing Technology. 2006;172(1):16-21.

5. Kim YS, Jain MK, Metzger DR. A finite element study of capstan friction test. AIP Conference Proceedings. 2004;712(1):22642269 .

6. Pereira MP, Duncan JL, Yan W, Rolfe BF. Contact pressure evolution at the die radius in sheet metal stamping. Journal of Materials Processing Technology. 2009;209(7):3532-3541.

7. Pereira MP, Yan W, Rolfe B. Contact pressure evolution and its relation to wear in sheet metal forming. Wear. 2008;265(1112):1687-1699.

8. Coubrough GJ, Alinger MJ, Van Tyne CJ. Angle of contact between sheet and die during stretch-bend deformation as determined on the bending-under-tension friction test system. Journal of Materials Processing Technology. 2002;130-131:69-75.

9. Hoffmann H, Nürnberg G, Ersoy-Nürnberg K, Herrmann G. A new approach to determine the wear coefficient for wear prediction of sheet metal forming tools. Production Engineering. 2007;1(4):357-363.
10. International Organization for Standardization. ISO 10275 Metallic materials - Sheet and strip - Determination of tensile strain hardening exponent. Switzerland: ISO; 2007.

11. International Organization for Standardization. ISO 10113 Metallic materials - Sheet and strip - Determination of plastic strain ratio. Switzerland: ISO; 2006.

12. International Organization for Standardization. ISO 6892-1 Metallic materials - Tensile testing - Part 1: Method of test at room temperature. Switzerland: ISO; 2016.

13. Andreasen JL, Olsson DD, Chodnikiewicz K, Bay N. Bending under tension test with direct friction measurement. Proceedings of the Institution of Mechanical Engineers, Part B: Journal of Engineering Manufacture. 2006;220(1):73-80.

14. Kim H, Sung JH, Sivakumar R, Altan T. Evaluation of stamping lubricants using the deep drawing test. International Journal of Machine Tools and Manufacture. 2007;47(14):2120-2132.

15. Han SS. Influence of tool geometry on friction behavior in sheet metal forming. Journal of Materials Processing Technology. 1997;63(1-3):129-133.

16. Jonasson M, Wihlborg A, Gunnarsson L. Analysis of surface topography changes in steel sheet strips during bending under tension friction test. International Journal of Machine Tools and Manufacture. 1998;38(5-6):459-467.

17. Wihlborg A, Gunnarsson L. A frictional study of uncoated EBT steel sheets in a bending under tension friction test. Wear. 2000;237(1):129-136.

18. Saha PK, Wilson WRD, Timsit RS. Influence of surface topography on the frictional characteristics of 3104 aluminum alloy sheet. Wear. 1996;197(1-2):123-129.

19. Hao S, Klamecki BE, Ramalingam S. Friction measurement apparatus for sheet metal forming. Wear. 1999;224(1):1-7.

20. Miguel VM, Coello J, Calatayud A, Manjabacas MC, Martínez A, Ferrer C. An approach to evaluation of sheet bending force under successive multiaxial stress condition. Journal of Materials Processing Technology. 2009;209(3):1588-1596.

21. Sube D. Possibilities and Results of Numeric and Experimental Simulation of Sheet Metal Forming Processes. In: Shaeffer L, editors. Proceedings of III Conferência Nacional de Conformação de Chapas; 2000 oct 24-26. Porto Alegre, Brazil. Porto Alegre: SENAFOR; 2000. p. 22-31. 